\title{
Multi-sensor fusion technology based tracking car
}

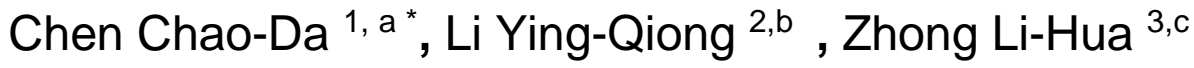

\author{
${ }^{1}$ Tianhe College of Guangdong Polytechnical Normal University, Guangzhou Guangdong China \\ ${ }^{1}$ Guangdong University of Technology, Guangzhou Guangdong China \\ ${ }^{2}$ Tianhe College of Guangdong Polytechnical Normal University, Guangzhou Guangdong China \\ ${ }^{3}$ Tianhe College of Guangdong Polytechnical Normal University, Guangzhou Guangdong China \\ agugu0769@126.com, b28477850@qq.com, candy448@126.com
}

Keywords: Single chip microcomputer; Infrared tube; Photoelectric sensor

\begin{abstract}
Multi sensor fusion technology of vehicle tracking is a set of environment perception, planning and decision-making, auxiliary driving level functions in an integrated system and is an important part of the intelligent transportation system. It has wide application prospects in military, civil, space development and other fields. The design of multi sensor fusion technology of vehicle tracking control system is researched, designed and implemented based on path planning of intelligent vehicle control system. At present, such devices have been applied to many aspects, such as archaeology, robots, entertainment and so on.Multi sensor fusion technology vehicle tracking is an important part of mobile robot, the design by real-time detection of each module of the sensor input signal using infrared to detect black line to achieve obstacle avoidance, through photoelectric sensors tracking the storage space larger as the main control chip, driving motor car used L298N chip, achieve automatic identification trolley line, the more effective control of the in run into obstacles can driving steering angle and tracking.
\end{abstract}

\section{Introduction}

With the continuous development of micro electronics technology, SCM is not only integrated level, it can be integrated into a chip CPU, memory, timer / counter, parallel and serial interface, A / D converter, D / A converter, and other circuits, and the volume is smaller and smaller, and it is easy to combine the computer technology and measurement control system.

Market in general multi sensor fusion technology tracking car can arm is adopted as the controller of the system, the advantage of this system is powerful, on-chip peripherals integration to engage in high density and improve the stability, the processing speed of the system is also very high, suitable for as the control core of large-scale real-time systems. And the speed of the car can not be too high, then the system will not be too high demand for processing information. If the program is used, it is bound to encounter a lot of problems in the control of unnecessary increase. The use of single-chip microcomputer as the core of the whole vehicle tracking system. With its control of smart car, both to achieve the desired performance indicators, but also a good operation to improve the operating environment of the car, and easy to use. For our control system, the core is how to achieve the automatic control of the car, for this, the microcontroller has a strong advantage - control is simple, convenient, fast, single-chip enough to meet the needs of our design.

In this paper, we research the multi sensor fusion technology integrated application and motor coordination control based on, and through single-chip microcomputer as the control core, motor driver L298N chip, using photoelectric sensor to detect black line to achieve the tracking, through infrared tube to achieve obstacle avoidance. 


\section{System plan determination and the selection of main components}

\subsection{System design}

Car tracking multi-sensor fusion technology is a branch of intelligent vehicle research. The use of single-chip microcomputer as the core of the whole vehicle tracking system. With its control of smart car, to achieve the desired performance indicators. It takes the wheel as a moving mechanism, can realize autonomous driving, so we call it the smart car. It has the basic characteristics of the robot, which is easy to program. Multi sensor fusion technology tracking car can through computer programming to achieve the car start and stop, driving direction and speed control, without human intervention. The operator can change its driving mode by modifying the computer program or some data of the smart car. This can be programmed to control and change the characteristics of the car driving mode is the biggest feature of smart car. The research purpose of the intelligent car control system is to make the car more autonomous. If any given car a path through the system, the car can get the system on the path of the data processing, and can be based on the displacement and angle information in accordance with the scheduled path.

Using single chip as the core, mainly including the tracking module, obstacle avoidance module, power supply module, motor drive module etc.,. By tracking sensors and obstacle avoidance sensors to collect environmental information to feedback to the CPU, through the main control, to control the motor running, so as to realize the tracking and obstacle avoidance, achieves the intelligent driving. In order to better accomplish the task of this design, we used L298N motor driver chip and the frame of the tricycle, the front of the left and right on both sides of the motor drive, two modulation in front motor rotating speed so as to achieve the purpose of steering control, rear wheel Vientiane, play a supporting role, and through software control, combined with the hardware architecture, thus solid line automatic tracking, obstacle avoidance function.

\section{2 tracking module}

Car tracking, we usually adopt the integrated CTRT5000 module, the sensor is an infrared photoelectric tube. This is a set of transmitter and receiver in one of the photoelectric sensor, Operating Voltage $5 \mathrm{~V}$, the black line output low level, the white line to output the high level, the detection distance can be adjusted according to our needs. But need to know is that the photoelectric sensor detection range is limited, it uses the non-contact detection method, the actual effective detection distance is $2 \sim 13 \mathrm{~mm}$.

\section{3 obstacle avoidance module}

Obstacle avoidance sensor module using infrared detection method, the sensor to ambient light adaptation ability, which has a pair of infrared transmitting and receiving tubes, tube launched a certain frequency infrared, when the direction detection encounter obstacles (reflector), infrared reflection back to be receiving tube receiving, after processing the comparator circuit, green indicator light, also signal output interface to output digital signal (a low level signal), through the potentiometer knob adjusts the detection distance, a range of effective distance $2 \sim 30 \mathrm{~cm}$, the working voltage of $3.3 \mathrm{v}-5 \mathrm{v}$. Detection range of the sensor can be through the potentiometer adjustment, with little interference, convenient assembly, easy to use, and can be widely applied to robot obstacle avoidance, obstacle avoidance of car, line count and black and white line tracking and so on numerous occasions.

\section{4 motor drive module}

The module uses ST L298N as the main driver chip, with strong driving ability, low heat, strong anti-interference ability is a high voltage, high current motor drive chip. The chip uses 15 pin package. The main features are: high working voltage, the highest operating voltage of up to $46 \mathrm{~V}$, the output current is big, the instantaneous peak current can reach $3 \mathrm{~A}$, the working current is $2 \mathrm{~A}$, the rated power is $25 \mathrm{~W}$. High voltage and high current full bridge driver with two $\mathrm{H}$ bridge can be used to drive DC motor and stepping motor, relay coil and other inductive loads. The standard logic level signal control, with two enable control, in the case of no input signal to allow or disable the device to work with a logic power input, so that the internal logic circuit part of the low voltage work; can be external detection resistor, will change the amount of feedback to control circuit. Using L298N chip drive 
motor, the chip can drive a two-phase stepper motor or four phase stepper motor, can also drive two DC motor.

The module can use the built-in 78M05 to power the power supply part, but in order to avoid the voltage regulator chip damage, when using the $12 \mathrm{~V}$ driver voltage, please use the external $5 \mathrm{~V}$ logic power supply. This module can improve the reliability by using the large capacity filter capacitor and the continued flow protection diode.

\section{3. program design}

\section{1 system programming process design}

This design by real-time detection of each module of the sensor input signal and tracking using photoelectric sensor to detect black line, through infrared tube to achieve obstacle avoidance, the collection of all the information is sent to the main processor and let the car to make the correct route.

Two DC motors can be driven by using a direct current drive. Were M1 and M2. Pin IN2, IN3 IN1, IN4 can be used for the input PWM pulse width modulation signal for motor speed control. (if there is no need for speed control can be two pin $5 \mathrm{~V}$, so that the motor work in the most high-speed state, both short cap short circuit to achieve the motor is more easy to reverse, the input signal terminal IN1 high level input IN2 low level, motor M1 is transferred. (if the signal is IN1 low, IN2 is high, the motor M1 is reversed.) Control the other motor is the same way, the input signal terminal IN3 high level, the input terminal IN4 low level, motor M2 is transferred. (PWM), the A signal is controlled by the M1, and the PWM signal is controlled by the B M2.

3.2 core code analysis

void main()

\{

pwm_init();

//Obstacle avoidance program

while $(\mathrm{b} 1==0\|\mathrm{~b} 2==0|| \mathrm{b} 3==0\| \mathrm{b} 4==0 \| \mathrm{b} 5==0)$

\{

scan_OA();

\}

//Start tracking program

while $(\mathrm{b} 1==1 \& \& \mathrm{~b} 2==1 \& \& \mathrm{~b} 3==1 \& \& \mathrm{~b} 4==1 \& \& \mathrm{~b} 5==1)$

\{

scan_line();

\}

\}

By program can know obstacle avoidance program priority in tracking program, so the encounter obstacles will first execute avoid obstacles, then continue to tracking. void scan_line()

\{

// Yanbian tracking

while $(\mathrm{x} 1==1 \& \& \mathrm{~b} 1==1 \& \& \mathrm{~b} 2==1 \& \& \mathrm{~b} 3==1 \& \& \mathrm{~b} 4==1 \& \& \mathrm{~b} 5==1)$

\{

pwm_set(0xff,0xff);

delay (150);

pwm_set(0x00,0xff);

delay $(600)$;

pwm_set(0xff,0xff);

delay(150);

\}

//Turn tracking

while $(\mathrm{x} 1==0 \& \& \mathrm{~b} 1==1 \& \& \mathrm{~b} 2==1 \& \& \mathrm{~b} 3==1 \& \& \mathrm{~b} 4==1 \& \mathrm{~b} 5==1)$ 


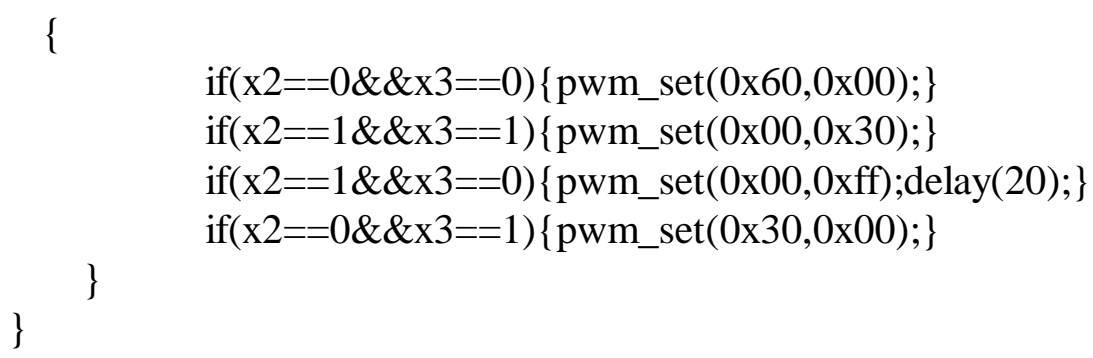

\section{Summary}

The design of the whole system is based on single-chip microcomputer, using a variety of sensors, the combination of software and hardware. This system can achieve the following functions: In the course of driving along the track of the preset track, the track can be automatically detected. If there is a deviation, can automatically correct, return to the preset track; when the car detects obstacles in front of the forward, can automatically avoid obstacles, from the barrier region through. The car through the barrier zone, automatically tracking.

Test results show that: from the view of the operation tracking effect is good, the car can from planning a complete track of any segment as a starting point, to finish the course. In addition, the car has the technology, knowledge, integration, can be used as an experimental device, to carry out experimental exercises.

\section{References}

[1] Chen Zhaoda. Based on the serial communication technology of the manipulator motion control [J]. manufacturing automation, 2013

[2] Wang Rong Ben. Overview of world intelligent vehicle research [J]. highway transportation technology, 2001 (10).

[3] Zhao. Futu sensor integrated circuit [M]. Handbook of Chemical Industry Press, 2004

[4] Jing Wong et al. Sensor module design of automatic tracing car [J]. modern electronic technology, 2008

[5] Sun Ying. Research on the control system of intelligent vehicle based on path planning [D]. Qiingdao University, 2007

[6] Zhao Lei. Based on wavelet decomposition of multi-source image fusion [J]. The coal,2010,9:189 $-191$

[7]Chen Chaoda.Temperature control in agricultural preservation application research[J].he International Journal of Advancements in Computing Technology, 2012,4(1):470-476

[8]Qiu-Ting Wang, Xiu-Lin Hu. New Kalman Filtering Algorithm for Passive-BD/SINS Integrated Navigation System Based on UKF, JCIT: Journal of Convergence Information Technology[J], vol.4, no.2, pp.102-107, 2009.

[9]Chen Chaoda. Intelligent Control System for Home Security[J]. Applied Mechanics and Materials , vol.65, pp.332-335, 2011.

[10] P.D. Liu, Y. Su.The extended TOPSIS based on trapezoid fuzzy linguistic variables[J], Journal of Convergence Information Technology, Vol. 5, No. 4, pp.38-53, 2010. 